\title{
Unravelling the ultrafast photodecomposition mechanism of dibenzoyl peroxide in solution by time-resolved IR spectroscopy
}

\author{
Received 29th November 2007, Accepted 2nd January 2008 \\ First published as an Advance Article on the web 31st January 2008 \\ DOI: $10.1039 /$ b718501d
}

Christian Reichardt,${ }^{a}$ Jörg Schroeder ${ }^{a b}$ Peter Vöhringer ${ }^{c}$ and Dirk Schwarzer ${ }^{* a}$

\begin{abstract}
The ultrafast photo-fragmentation of dibenzoyl peroxide (DBPO) is studied using femtosecond UV excitation at $266 \mathrm{~nm}$ and mid-infrared broadband probe pulses to elucidate the dissociation mechanism. With the help of ${ }^{13} \mathrm{C}$-labeled DBPO it was possible to unambiguously assign transient IR bands in the fingerprint region to the benzoyloxy radical. Our experiments show that the fragmentation is controlled by the $\mathrm{S}_{1}$-lifetime of DBPO and within $0.4 \pm 0.2 \mathrm{ps}$ leads to a benzoyloxy/phenyl radical pair plus $\mathrm{CO}_{2}$ via concerted bond breakage of the $\mathrm{O}-\mathrm{O}$ and the phenyl-C(carbonyl) bond. $20 \%$ of the radical pairs geminately recombine to phenyl benzoate on a timescale of $70 \mathrm{ps}$.
\end{abstract}

\section{Introduction}

Dibenzoyl peroxide (DBPO) is a widely used initiator of freeradical polymerizations in solution whose dissociation can be induced photochemically or by thermal activation. Whereas the thermal decomposition is well understood and proceeds via bond breakage of the $\mathrm{O}-\mathrm{O}$ bond yielding benzoyloxy radicals which subsequently either decarboxylate on a microsecond time scale to form phenyl radicals (pathway 1 in Scheme 1) or are scavenged by reaction with the solvent or appropriate substrates, ${ }^{1}$ the primary steps of fragmentation after UV excitation of DBPO are not completely understood. Early chemical trapping experiments ${ }^{2}$ showed that the photodissociation not only produces benzoyloxy radicals (which later were characterized by their UV-Vis ${ }^{3}$ and $\mathrm{EPR}^{4,5}$ spectra), but also in a two-bond cleavage phenyl radicals and $\mathrm{CO}_{2}$ as expressed by pathway 2 in Scheme 1. The importance of pathway 2 is supported by the observation of the photoproduct phenyl benzoate formed by geminate recombination of the benzoyloxy-phenyl radical pair (see ref. 6 and references cited therein).

Consistent with pathway 2 are results from picosecond UVpump/IR-probe spectroscopy indicating $\mathrm{CO}_{2}$ formation within the experimental time resolution of about $10 \mathrm{ps}^{7}$ According to this study the nascent $\mathrm{CO}_{2}$ molecules possess large excess energy corresponding to an initial vibrational temperature of $2300 \mathrm{~K}$.

Recently, based on femtosecond pump-probe experiments employing $267 \mathrm{~nm}$ excitation, Abel et al. ${ }^{8}$ suggested an alternative model in which they assigned the transients in the visible to near-IR spectral range that decayed to a constant offset on a picosecond timescale to hot benzoyloxy radicals.

\footnotetext{
${ }^{a}$ Abteilung Spektroskopie und Photochemische Kinetik, Max-PlanckInstitut für Biophysikalische Chemie, 37070 Göttingen, Germany. E-mail:dschwar@gwdg.de

${ }^{b}$ Institut für Physikalische Chemie, Georg-August-Universität Göttingen, Tammannstraße 6, 37077 Göttingen, Germany

${ }^{c}$ Institut für Physikalische und Theoretische Chemie, Rheinische Friedrich-Wilhelms-Universität Bonn, Wegeler Str. 12, 53115 Bonn, Germany
}

According to their model, DBPO photofragmentation initially follows pathway 1 with $\mathrm{O}-\mathrm{O}-$ bond scission being faster than $200 \mathrm{fs}$, yielding highly vibrationally excited benzoyloxy radicals. A fraction $f$ of these radicals rapidly decomposes to phenyl plus $\mathrm{CO}_{2}$ (dashed arrow in Scheme 1), while the remaining population by vibrational energy relaxation cools down to thermal equilibrium and subsequently decarboxylates on a microsecond timescale. This analysis, however, rests upon the correct assignment of transient electronic spectra in the visible and near-IR. As the absorption bands of intermediates including electronically excited states are broad or not known, extensive overlap of spectra may render the definitive assignment to certain species difficult. In the picosecond time range the analysis may be even more complicated by spectral shifts and band narrowing caused by vibrational relaxation of hot intermediates.

As vibrational absorption bands are narrower than electronic spectra, femtosecond broadband IR spectroscopy can provide additional information on complex photoreactions. In cases where the IR spectra are not known quantum chemical calculations offer the opportunity to compare experimental and theoretical spectra and thus identify the relevant intermediates. Using this approach we recently discovered that the decarboxylation rate of the arylperoxocarbonates $\mathrm{R}-\mathrm{O}-\mathrm{C}(\mathrm{O}) \mathrm{O}-\mathrm{OBu}^{\mathrm{t}}(\mathrm{R}=$ naphthyl, phenyl) is limited by the $\mathrm{S}_{1}$-lifetime of the photo-excited peroxide and that an intermediate aryloxycarbonyloxy radical cannot be detected. ${ }^{9}$ In this paper we present a detailed analysis of the primary steps of the photodecomposition of DBPO. We focus on the question whether in the fragmentation all bonds break sequentially as suggested by femtosecond UV/Vis pump-probe

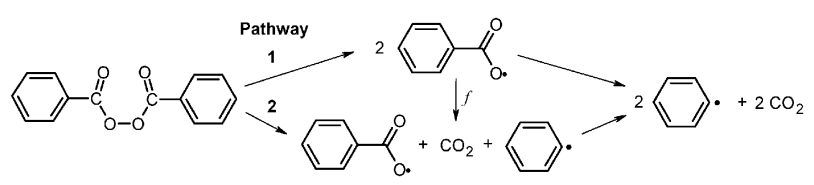

Scheme 1 Reaction pathways of dibenzoyl peroxide (DBPO) decomposition. 
experiments ${ }^{8}$ or whether possibly concerted bond cleavage is involved. Also, the geminate recombination of the benzoyloxy-phenyl radical pair should be detectable by our approach. Unambiguous assignment of IR bands was achieved by additional experiments with ${ }^{13} \mathrm{C}$-labeled DBPO molecules.

\section{Experimental technique}

The femtosecond infrared experiments were carried out with 1-3 mM solutions of DBPO dissolved in $\mathrm{CD}_{3} \mathrm{CN}, \mathrm{CD}_{2} \mathrm{Cl}_{2}$ (Deutero $\mathrm{GmbH}, 99.6 \%$ deuteration grade), or $n$-heptane. Sample solutions were excited in a stainless steel flow cell equipped with $1 \mathrm{~mm}$ thick $\mathrm{CaF}_{2}$ windows (path length inside the cell $0.6-1.0 \mathrm{~mm}$ ) by the third harmonic of a home-built $1 \mathrm{kHz}$ Ti:sapphire laser system (wavelength $267 \mathrm{~nm}$, pulse duration $100 \mathrm{fs}$, pulse energy $1-2 \mu \mathrm{J})$. Tunable infrared pulses were generated by difference frequency mixing of signal and idler pulses from an optical parametric amplifier ${ }^{10,11}$ (pulse duration $100 \mathrm{fs}$, pulse energy $0.5-1 \mu \mathrm{J}$, bandwidth of $200 \mathrm{~cm}^{-1}$ ). The IR pulses were split into a probe and a reference beam; at the sample the probe beam was superimposed on the pump beam. The relative plane of polarization of both pulses was adjusted to $54.7^{\circ}$ to avoid signal contributions arising from rotational relaxation of molecules. After passing the sample reference and probe beam were spectrally dispersed with a polychromator and independently imaged on a liquid-nitrogen cooled $\mathrm{HgCdTe}$ detector $(2 \times 32$ pixels $)$. The whole pump-probe setup was purged with dry nitrogen to avoid spectral and temporal distortion of the IR pulses by $\mathrm{CO}_{2}$ and water absorptions in air.

DBPO (Fluka, water content $25 \%$ ) was dried by repeatedly dissolving a sample in dry methanol and subsequently evapor- ating the solvent under reduced pressure. ${ }^{13} \mathrm{C}$-labelled DBPO $\left(\left(\mathrm{C}_{6} \mathrm{H}_{5}{ }^{13} \mathrm{CO}_{2}\right)_{2}\right)$ was synthesised from the corresponding benzoic acid $\left(\mathrm{C}_{6} \mathrm{H}_{5}{ }^{13} \mathrm{CO}_{2} \mathrm{H}\right.$, Aldrich $)$ adopting the imidazolid method. $^{12}$

To verify the structural and vibrational properties of DBPO and the corresponding radicals quantum chemical calculations using density functional theory (DFT) were performed with the ORCA program package ${ }^{13,14}$ developed by Neese. To enhance the efficiency of the calculations at no significant loss of accuracy, the resolution-of-identity (RI) approximation was invoked. ${ }^{15}$ Since this procedure requires a non-hybrid functional, we have chosen the Becke-Perdew functional, BP86. ${ }^{16,17}$ The Ahlrichs' triple- $\zeta$ valence basis set, ${ }^{18}$ TZVPP, with three sets of polarization functions on all atoms was applied, to which diffuse functions from Dunning's correlation-consistent polarized triple- $\zeta$ basis set ${ }^{19}$ could be added (hence, aug-TZVPP). In addition, the RI-approximation requires an auxiliary Coulomb fitting basis $(\mathrm{TZV} / \mathrm{J})$ for the triple- $\zeta$ valence basis set, which was taken from the TURBOMOLE library. ${ }^{20}$ Structure optimizations with and without geometrical constraints were carried out in redundant internal coordinates using analytical gradients, whose double-sided numerical differentiation yielded harmonic vibrational frequencies. Following the recommendations by Neugebauer and Hess ${ }^{21}$ for calculations at the RI-DFT/TZVPP level of theory, a correction factor of 1.005 for conversion from harmonic to fundamental vibrational frequencies was used.

\section{Results and discussion}

Fig. 1(a) shows linear absorption spectra of DBPO and ${ }^{13} \mathrm{C}$ DBPO in $\mathrm{CD}_{3} \mathrm{CN}$. For both compounds the $\mathrm{CO}$ stretching
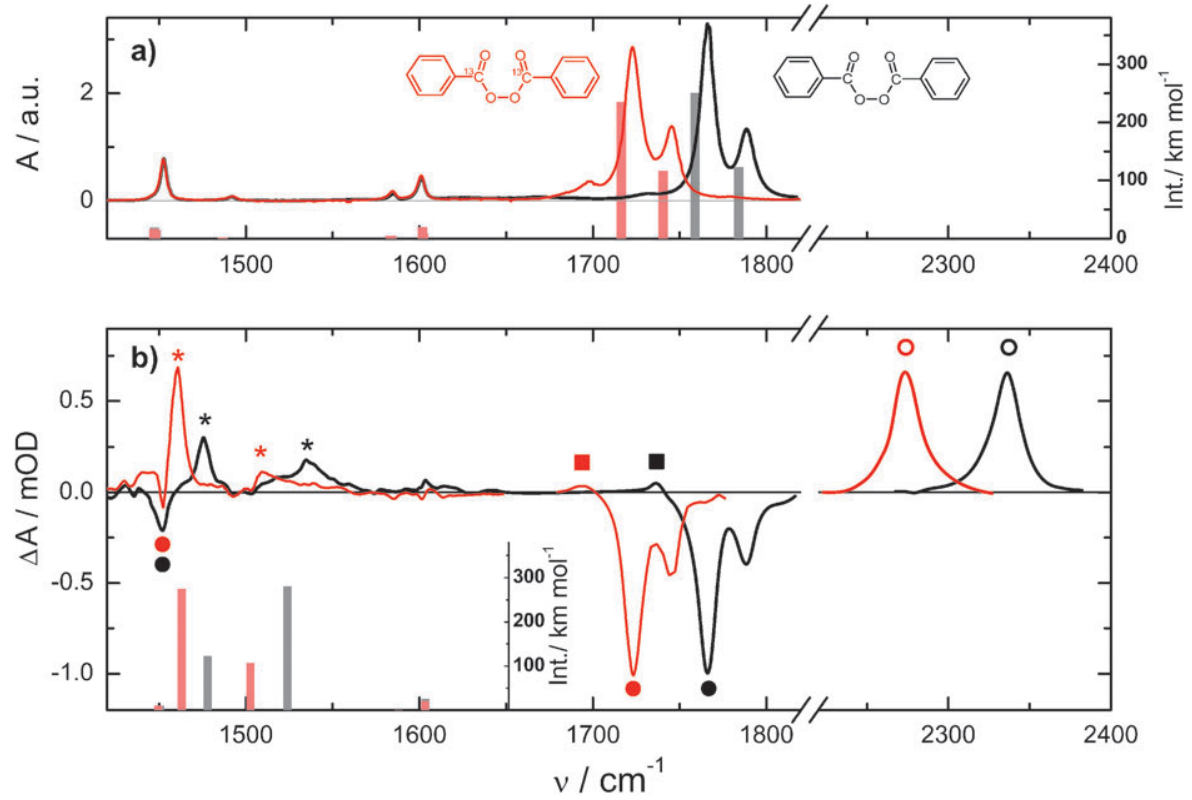

Fig. 1 (a) Linear absorption spectra of DBPO (black) and ${ }^{13} \mathrm{C}-\mathrm{DBPO}$ (red) in $\mathrm{CD}_{3} \mathrm{CN}$ (bars indicate calculated frequencies and intensities); (b) transient difference spectra $1.3 \mathrm{~ns}$ after $267 \mathrm{~nm}$ excitation of DBPO (black) and ${ }^{13} \mathrm{C}$-DBPO (red) in $\mathrm{CD}_{3} \mathrm{CN}\left(\right.$ at $2220-2380 \mathrm{~cm}^{-1}$ spectra were recorded in $n$-heptane, because $\mathrm{CD}_{3} \mathrm{CN}$ is opaque in this range); symbols indicate bleaching of DBPO (filled circles) and formation of the products $\mathrm{CO}_{2}$ (open circles), benzoyloxy radical (stars), and phenyl benzoate (squares); the inset shows calculated frequencies and intensities of the benzoyloxy radical. 
band is split into two components arising from symmetric (DBPO: $1789 \mathrm{~cm}^{-1} ;{ }^{13} \mathrm{C}-\mathrm{DBPO}: 1746 \mathrm{~cm}^{-1}$ ) and antisymmetric (1766 and $1723 \mathrm{~cm}^{-1}$, respectively) combination vibrations of the two carbonyl groups. Our DFT calculations indicate a decisive influence of the COOC dihedral angle on frequency and IR intensity ratio of both $\mathrm{CO}$ stretching vibrations. For the minimum energy structure of DBPO with $\varphi_{\mathrm{COOC}}=87^{\circ}\left(\text { crystal structure data give } \varphi_{\mathrm{COOC}}=91^{\circ}\right)^{22}$ frequencies and IR intensity ratios (bars in Fig. 1(a)) are in agreement with the measured spectra. Also the experimental isotope shift of $43 \mathrm{~cm}^{-1}$ is nicely reproduced by the calculations $\left(42.5 \mathrm{~cm}^{-1}\right)$. Weaker red-shifted absorption bands in the CO-stretching region are probably due to other conformers. The absorption bands at 1453, 1492, 1585 and $1601 \mathrm{~cm}^{-1}$ correspond to $\mathrm{CH}$ bending and $\mathrm{CC}$ stretching vibrations of the phenyl ring and hence are almost unaffected by ${ }^{13} \mathrm{C}$-labeling of the carbonyl groups. Using Wilson's notation of benzene these modes can be assigned to $19 \mathrm{~b}, 19 \mathrm{a}, 8 \mathrm{~b}$ and $8 \mathrm{a}$, respectively.

In Fig. 1(b) transient difference spectra recorded $1.3 \mathrm{~ns}$ after $\mathrm{UV}$ excitation of DBPO and ${ }^{13} \mathrm{C}$-DBPO in $\mathrm{CD}_{3} \mathrm{CN}$, respectively, are presented for the frequency range $1420-1820 \mathrm{~cm}^{-1}$. Because of the limited bandwidth of the IR probe pulse they were constructed from several overlapping spectra of $\sim 100$ $\mathrm{cm}^{-1}$ width. The estimated error of the relative amplitudes of spectral features arising from this procedure is estimated to be $<25 \%$. At $2220-2400 \mathrm{~cm}^{-1}$ where the solvent $\mathrm{CD}_{3} \mathrm{CN}$ is opaque separate transient spectra of DBPO and ${ }^{13} \mathrm{C}-\mathrm{DBPO}$ in $n$-heptane are shown. This part of the spectrum is not to scale.

The transient spectra show negative peaks caused by bleaching of the peroxide and positive absorptions due to the formation of photoproducts. Most prominent is the bleaching of the $\mathrm{CO}$ stretching bands but also phenyl ring vibrations at $1453 \mathrm{~cm}^{-1}$ clearly show the disappearance of DBPO and ${ }^{13} \mathrm{C}$ DBPO, respectively, as indicated by full circles. The strongest increase of absorption arises from the asymmetric stretching vibration of newly formed $\mathrm{CO}_{2}$ at $2337 \mathrm{~cm}^{-1}$ and ${ }^{13} \mathrm{CO}_{2}$ at $2273 \mathrm{~cm}^{-1}$, respectively (open circle in Fig. 1(b)). In the fingerprint region strong and sharp bands at 1476 (DBPO) and $1461 \mathrm{~cm}^{-1}\left({ }^{13} \mathrm{C}\right.$-DBPO) and, additionally, weaker but broader absorptions at 1535 (DBPO) and $1508 \mathrm{~cm}^{-1}\left({ }^{13} \mathrm{C}\right.$ DBPO) appear as marked by asterisks. Because of the pronounced isotope shift of $15 \mathrm{~cm}^{-1}$ for the sharp and $27 \mathrm{~cm}^{-1}$ for the broad peaks we attribute these bands to the benzoyloxy radical. Infrared spectra of this radical have not been published so far. Our DFT calculations of the unlabeled radical yield strong IR active vibrations at 1478 and $1524 \mathrm{~cm}^{-1}$ with an intensity ratio of about $1: 2.3$ as demonstrated in the inset of Fig. 1(b). Upon labelling these vibrations shift down to 1463 and $1503 \mathrm{~cm}^{-1}$, respectively, where the intensity ratio is reversed to $2.5: 1$. This is in excellent agreement with the measurements such that the asterisked bands unambiguously can be assigned to the benzoyloxy radical. A closer analysis of the calculations reveal that both strongly IR active modes involve combinations of $\mathrm{OCO}$ and phenyl- $\mathrm{CH}$ bending (mode 19a in Wilson notation) vibrations of the benzoyloxy radical. For the low-frequency mode the two motions are in phase whereas for the high-frequency mode they are out of phase.
Finally, Fig. 1(b) shows weak absorptions (squares) at 1737 (DBPO) and $1694 \mathrm{~cm}^{-1}\left({ }^{13} \mathrm{C}\right.$-DBPO), $30 \mathrm{~cm}^{-1}$ red-shifted to the antisymmetric $\mathrm{CO}$ stretching vibration of the respective parent molecule. Obviously, a new compound has been formed where at least one carbonyl group of the peroxide has been preserved. In the case of DBPO the frequency of the new band coincides exactly with the $\mathrm{CO}$ stretching vibration of phenyl benzoate dissolved in $\mathrm{CD}_{3} \mathrm{CN}$. Thus, $1.3 \mathrm{~ns}$ after photodissociation of DBPO we have clear evidence for phenyl benzoate which within this time range can only be formed by geminate recombination of the benzoyloxy-phenyl radical pair. The phenyl radical itself is not detectable in our experiment because its absorptions are too weak. ${ }^{23}$

Further evidence supporting the assignment of the $\mathrm{CO}$ stretching absorption to phenyl benzoate is provided from the time evolution of these bands presented in Fig. 2 for the photodissociation of DBPO in $n$-heptane. Immediately upon excitation by the UV pump pulse, CO stretching bands in the electronic ground and first excited state of DBPO appear as a superposition of bleach and absorption, respectively (Fig. 2(a)). With increasing pump-probe delay the excited state population decays and only the ground state bleach remains. An exponential fit to the integrated band intensity (inset in Fig. 2(a)) gives a $S_{1}$ lifetime of $0.4 \pm 0.2$ ps. Then, on a longer
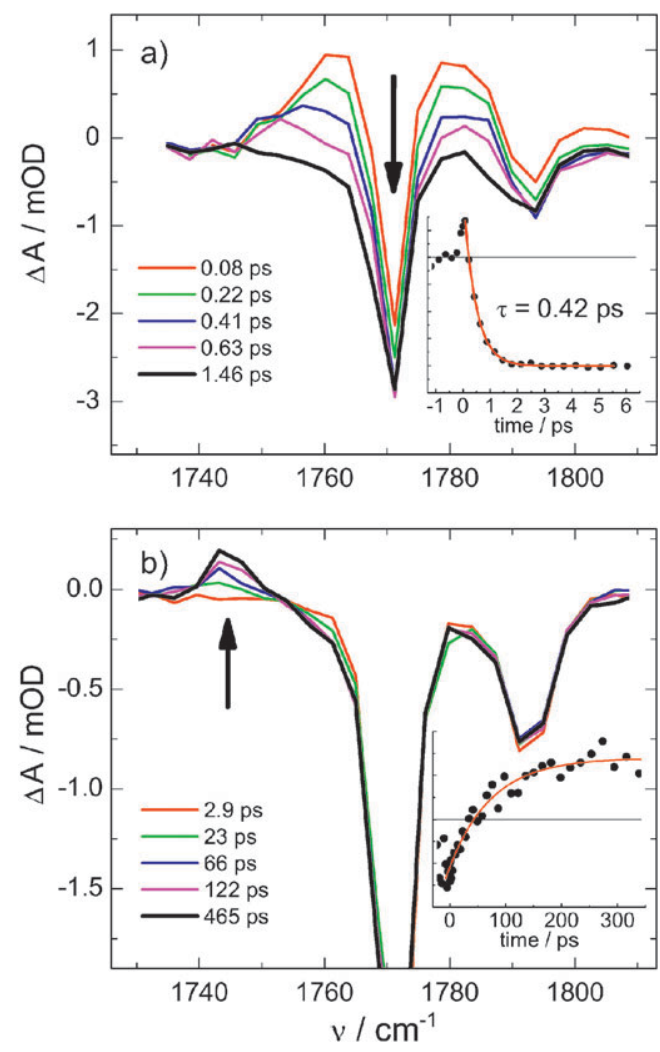

Fig. 2 Transient difference spectra of the CO stretch absorption band of DBPO in $n$-heptane recorded at selected pump-probe delay times (arrows indicate chronology); (a) at early times the $\mathrm{S}_{1}$ decay of DBPO is monitored (the inset shows the temporal evolution of the integrated band intensity, exponential fit gives a time constant of $0.42 \pm 0.15 \mathrm{ps}$ ); (b) at later times formation of the recombination product phenyl benzoate is observed (inset: band intensity around $1745 \mathrm{~cm}^{-1}$; red curve: exponential fit gives a time constant of $75 \pm 10 \mathrm{ps}$ ). 
timescale the new $\mathrm{CO}$ stretching band of phenyl benzoate emerges at $1745 \mathrm{~cm}^{-1}$ (Fig. 2(b)). Note, that the slightly blueshifted absorption in $n$-heptane with respect to $\mathrm{CD}_{3} \mathrm{CN}$ (Fig. 1(b)) is in complete agreement with the corresponding stationary spectra of phenyl benzoate. The time constant of $75 \pm$ $10 \mathrm{ps}$ derived from exponentially fitting the temporal evolution of the band integral (inset in Fig. 2(b)) is typical for geminate recombination.

Fig. 3 shows the formation of $\mathrm{CO}_{2}$ monitored at the $\nu_{3}$ asymmetric stretching vibration following photoexcitation of DBPO in $n$-heptane. The contour plot demonstrates that $\mathrm{CO}_{2}$ is formed vibrationally hot as initially the spectrum is broad and red-shifted with respect to the $(0,0,0) \rightarrow(0,0,1)$ transition at $2337 \mathrm{~cm}^{-1}$. This behavior is in agreement with earlier studies. ${ }^{7,9}$ The excess energy originates from the structural relaxation associated with the transformation of the bent $\mathrm{OCO}$ moiety into the linear $\mathrm{CO}_{2}$ molecule. According to molecular dynamics calculations the subsequent energy transfer to the bath observed in Fig. 3 involves mainly the strongly coupled bending and symmetric stretching vibrations of $\mathrm{CO}_{2}$, whereas the symmetric stretching mode relaxes on a much longer timescale. ${ }^{24,25}$ In contrast to the solvent $\mathrm{CCl}_{4}$ where the $\nu_{3}$ spectrum of vibrationally excited $\mathrm{CO}_{2}$ is characterized by a $12.5 \mathrm{~cm}^{-1}$ progression caused by anharmonic coupling to the bending vibration ${ }^{9}$ this feature is covered by the larger intrinsic line width of $14 \mathrm{~cm}^{-1}$ in $n$-heptane (see right panel of Fig. 3) as compared to $\mathrm{CCl}_{4}\left(6 \mathrm{~cm}^{-1}\right)$. Nevertheless, the individual spectra can be modeled assuming a Boltzmann distribution within the $\mathrm{CO}_{2}$ vibrational manifold to determine the evolution of the vibrational temperature as described previously. ${ }^{9}$ The time constant of the temperature decay of $\tau_{T}=(145 \pm 15)$ ps turns out to be in complete agreement with the corresponding value determined in the decarboxylation of peroxycarbonates. ${ }^{9}$ However, the initial temperature of $T_{0}=$ $(1500 \pm 100) \mathrm{K}$ is significantly below $2500 \mathrm{~K}$ found for the peroxycarbonates which corroborates earlier studies. ${ }^{7}$ In the upper panel and the inset of Fig. 3 the growth of the $\mathrm{CO}_{2}$ concentration derived from the integral $\nu_{3}$ band intensity is plotted. The rise time of $0.4 \pm 0.2 \mathrm{ps}$ is within experimental error identical to the time constant for the $\mathrm{S}_{1}$ decay of DBPO, i.e. the decarboxylation of DBPO is limited by the $\mathrm{S}_{1}$-lifetime similar to the mechanism found for the photofragmentation of aromatic peroxycarbonates. ${ }^{9}$ No further change of the $\mathrm{CO}_{2}$ concentration is observed up to $500 \mathrm{ps}$.

After UV excitation of DBPO also the benzoyloxy radical is formed on a subpicosecond timescale as shown in the contour diagram of Fig. 4 for the ${ }^{13} \mathrm{C}$-labeled compound dissolved in $\mathrm{CD}_{3} \mathrm{CN}$. The absorption of the radical at $1461 \mathrm{~cm}^{-1}$ is superimposed by bleaching of the sharp $\mathrm{CH}$ bending vibration of the parent molecule at $1453 \mathrm{~cm}^{-1}$. Nevertheless, Fig. 4 clearly evidences that initially the radical band is broad and narrows within several tens of ps. As for $\mathrm{CO}_{2}$ this is a clear signature of vibrational energy relaxation of a hot species. The absorption reaches a maximum at 50 ps and, subsequently, slightly decreases on a 100 ps time scale. For analyzing the temporal evolution of the relative radical concentration the band intensity was calculated after subtracting the bleach spectrum of the parent molecule at every time step. The result is plotted in the upper panel of Fig. 4 and shows that after formation the radical concentration decreases by about $15 \%$ with a time constant of $80 \pm 20$ ps which coincides with the time constant observed for the geminate recombination of the benzoyloxyphenyl radical pair to phenyl benzoate. Note, that parallel to vibrational energy relaxation and decline of the $1461 \mathrm{~cm}^{-1}$

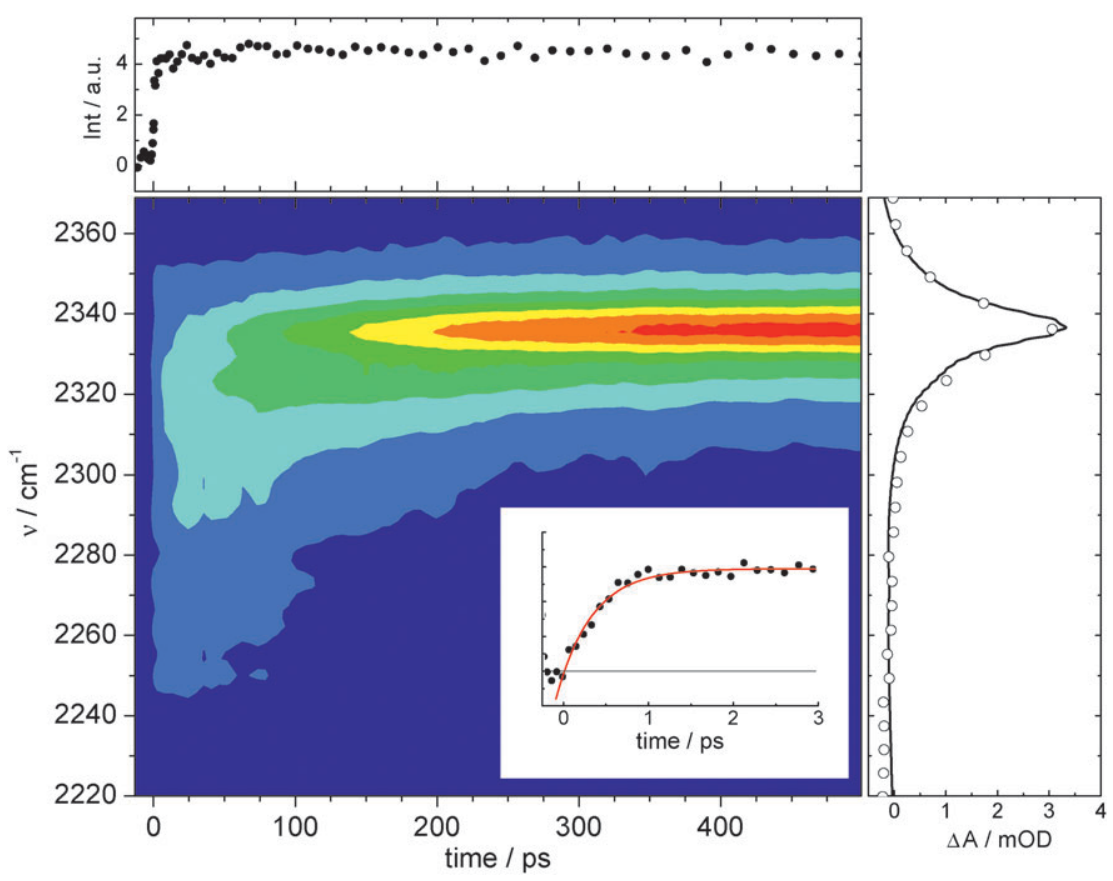

Fig. 3 Spectral evolution of the $\nu_{3}$ absorption band of $\mathrm{CO}_{2}$ produced in the photodissociation of DBPO in $n$-heptane; right panel: comparison of transient spectrum at $500 \mathrm{ps}$ pump-probe delay (points) with the linear absorption spectrum of $\mathrm{CO}_{2}$ dissolved in $n$-heptane (black line); upper panel: integrated band intensity; inset: short time evolution of the integrated band intensity (red curve: exponential fit to the data with time constant of $0.4 \pm 0.2 \mathrm{ps})$. 


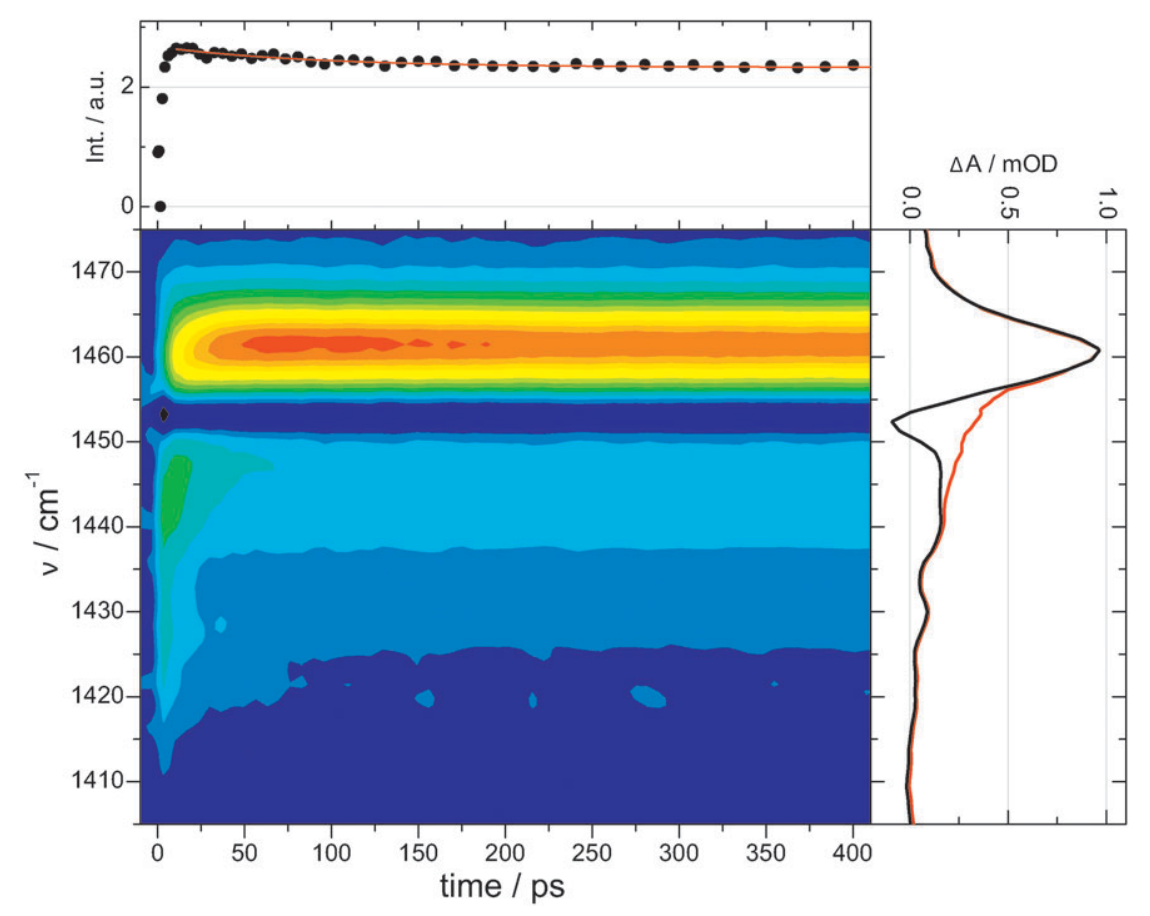

Fig. 4 Spectral evolution of the $1461 \mathrm{~cm}^{-1}$ absorption band of the benzoyloxy radical formed after photodissociation of ${ }^{13} \mathrm{C}-\mathrm{DBPO}$ in $\mathrm{CD}_{3} \mathrm{CN}$; right panel: transient spectrum at 500 ps (black) and after subtraction of the bleach component of the parent molecule (red); upper panel: integrated band intensity subtracting the bleach of the parent molecule (red curve: exponential fit with time constant $80 \pm 20 \mathrm{ps)}$.

band no increase of the $\mathrm{CO}_{2}$ concentration is detectable. This indicates that the decrease of the benzoyloxy radical concentration is mainly caused by recombination with phenyl radicals.

Quantum yields of $\mathrm{CO}_{2}$ formation and geminate recombination can be estimated from a comparison of transient spectra with linear CO-stretch band extinction coefficients. The upper left part of Fig. 5 shows extinction coefficients of DBPO (black) and phenyl benzoate (blue) in the CO stretching region. Underneath we give the difference spectra measured at the $\mathrm{CO}$ stretching band frequency after UV excitation of DBPO (thick black lines) scaled to the extinction coefficient

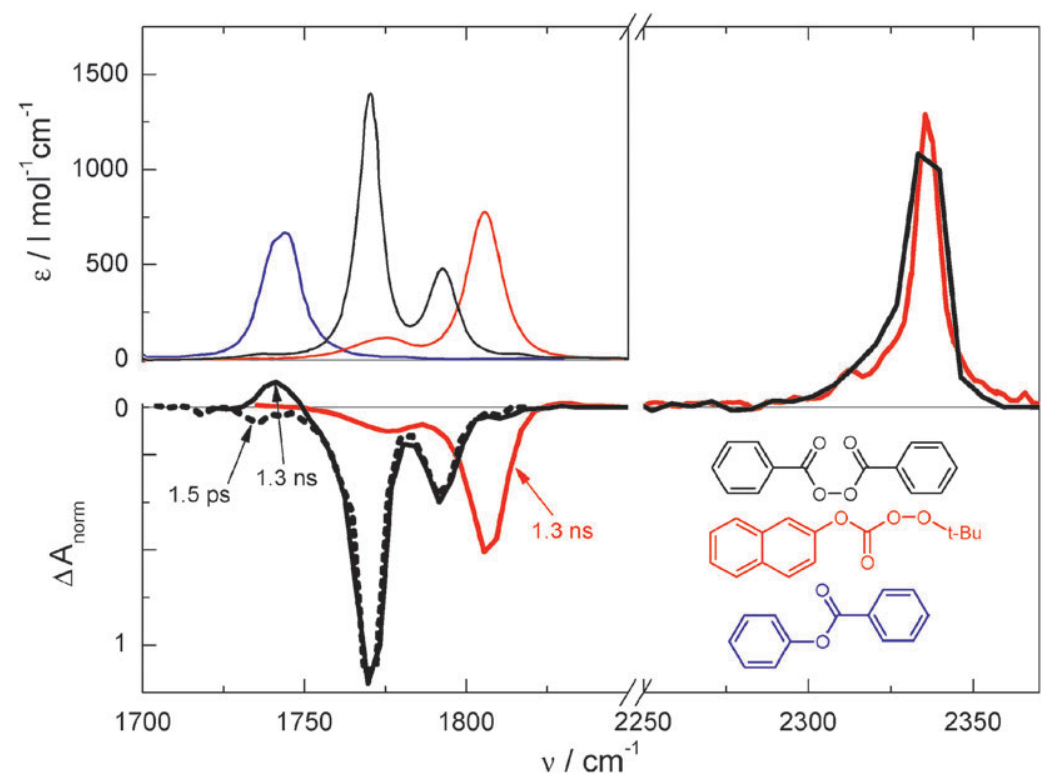

Fig. 5 Quantum yields of phenyl benzoate and $\mathrm{CO}_{2}$ after photodissociation of DBPO in $\mathrm{CCl}_{4}$. The upper left part shows extinction coefficients of the CO-stretch absorption band for DBPO (black), phenyl benzoate (blue), and tert-butyl-2-naphthylperoxycarbonate (TBNC, red); corresponding molecular structures are plotted in the same colour. Underneath pump-probe difference spectra of DBPO (thick black lines) and TBNC ${ }^{9}$ (thick red line) normalized to the respective extinction coefficient are presented. From the difference of the DBPO transients at 1.5 ps and $1.3 \mathrm{~ns}$ a phenyl benzoate quantum yield of $25 \%$ can be calculated. The amplitudes of the $\nu_{3}$ asymmetric stretching bands suggest that similar concentrations of $\mathrm{CO}_{2}$ are produced in the photodissociations of TBNC and DBPO. 
of DBPO. By scaling the difference between the $1.5 \mathrm{ps}$ and the $1.3 \mathrm{~ns}$ transient at $1742 \mathrm{~cm}^{-1}$ with the phenyl benzoate extinction coefficient we obtain a quantum yield of $25 \%$ for geminate recombination of the benzoyloxy/phenyl radical pair which is in good agreement with the drop of the benzoyloxy concentration derived from Fig. 4.

The $\mathrm{CO}_{2}$ quantum yield of DBPO photodissociation were determined by using tert-butyl-2-naphthylperoxycarbonate (TBNC) as a reference for which the decarboxylation quantum yield is close to unity. ${ }^{9}$ The extinction coefficient of TBNC is shown in the upper left part of Fig. 5 as a red curve. The corresponding pump-probe spectrum normalized to the extinction coefficient is also plotted in Fig. 5 (thick red line) and at $2336 \mathrm{~cm}^{-1}$ shows the same $\mathrm{CO}_{2}$ concentration as obtained from the decarboxylation of DBPO. In other words, the $\mathrm{CO}_{2}$ quantum yield of the primary DBPO photodissociation also is about unity.

\section{Summary and conclusion}

Our results on the DBPO photo-fragmentation can be summarized as follows:

(1) The $S_{1}$-lifetime of the photo excited DBPO molecule is extremely short and averages to $0.4 \pm 0.2 \mathrm{ps}$ in the solvents studied $\left(\mathrm{CCl}_{4}, \mathrm{CH}_{2} \mathrm{Cl}_{2}, \mathrm{CD}_{3} \mathrm{CN}, n\right.$-heptane)

(2) $\mathrm{CO}_{2}$ and benzoyloxy radicals are formed with the same rate as the electronically excited DBPO molecule decays.

(3) The quantum yield of $\mathrm{CO}_{2}$ in the direct dissociation of DBPO is $\Phi_{\mathrm{CO}_{2}}=1.0 \pm 0.2$ and remains unchanged up to $1 \mathrm{~ns}$.

(4) During this time the benzoyloxy radical concentration decreases by $15-25 \%$ with a time constant of $(70 \pm 10)$ ps. This decay is accompanied by corresponding formation of the geminate recombination product phenyl benzoate.

The dissociation mechanism emerging from these findings is summarized in Scheme 2. It definitely involves a concerted two-bond cleavage into phenyl, benzoyloxy and $\mathrm{CO}_{2}$ with a rate constant limited by the $\mathrm{S}_{1}$-lifetime of DBPO.

The transients in the visible spectral range observed previously ${ }^{8}$ after photo-excitation of DBPO also rapidly decayed on a subpicosecond to picosecond timescale as illustrated in Fig. 6. The instantaneously formed main peak between 565 and $770 \mathrm{~nm}$ disappears with a dominant time constant of $(0.45$ $\pm 0.10) \mathrm{ps}$, identical to the $\mathrm{S}_{1}$-lifetime found in this IR-study. The original view was that this transient has to be assigned to vibrationally hot benzoyloxy radicals decomposing into phenyl plus $\mathrm{CO}_{2}$, while the preceding first step in the reaction mechanism, breakage of only the peroxide $\mathrm{O}-\mathrm{O}$ bond, could not be observed in the experiment. Fig. 4 shows that, indeed, the benzoyloxy radical is formed vibrationally hot and cools down within the first $10-20$ ps, but this process is not

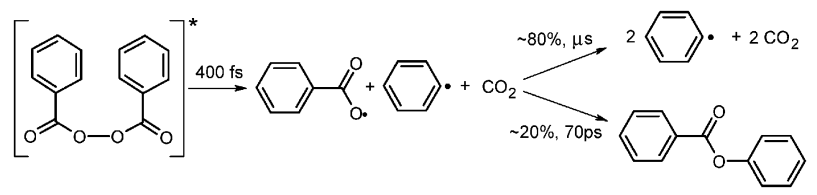

Scheme 2 Timescales and yields of the photofragmentation of DBPO.

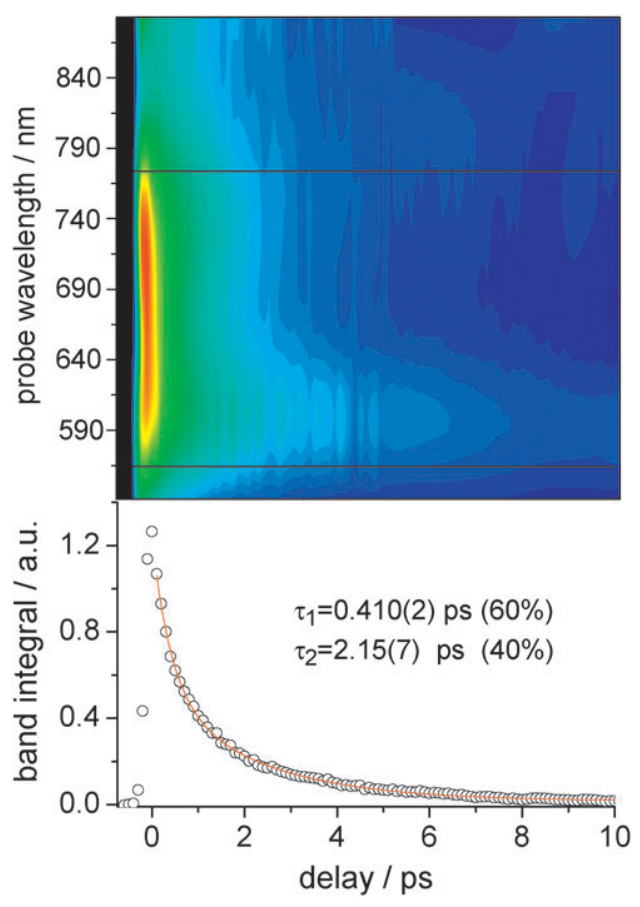

Fig. 6 Spectral evolution of the main transient absorption band in the visible after UV-excitation of DBPO in propylene carbonate solution. The bottom graph shows the decay of the band integral between the wavelength limits indicated by the horizontal lines in the contour plot.

connected with a fragmentation as the radical concentration does not exhibit a fast decay. Also, the $\mathrm{CO}_{2}$ concentration is constant during this period. Therefore, the strong subpicosecond transient in the visible region cannot be attributed to the benzoyloxy radical. Instead, we reassign them to the $\mathrm{S}_{n} \leftarrow \mathrm{S}_{1}$ absorption of DBPO. On the other hand, we attribute a second component of the transient absorption decay in the visible with a time constant of about $(3 \pm 1)$ ps to spectral narrowing as a consequence of vibrational cooling of the benzoyloxy radical whose room-temperature absorption spectrum extends from its maximum in the near IR to about $620 \mathrm{~nm}$.

The only reaction consuming benzoyloxy radicals within the first nanosecond is geminate recombination with the phenyl radical. The yield is only weakly dependent on the solvent and on average amounts to $\sim 20 \%$. This value is in complete agreement with stationary measurements of the phenyl benzoate quantum yield. ${ }^{6}$ The decarboxylation of the remaining benzoyloxy radical population is a thermally activated process and takes microseconds. ${ }^{1}$

\section{Acknowledgements}

The authors are grateful to Jens Schimpfhauser and Jürgen Bienert for the preparation and purification of ${ }^{13} \mathrm{C}$-labelled DBPO. We also thank Jürgen Troe for continuous support of this work. 


\section{References}

1 K. Fujimori, Diacyl peroxides, in Organic Peroxides, ed. W. Ando, Wiley, New York, 1992, p. 319.

2 J. C. Bevington and T. D. Lewis, Trans. Faraday Soc., 1958, 54, 1340.

3 J. Chateauneuf, J. Lusztyk and K. U. Ingold, J. Am. Chem. Soc., 1988, 110, 2886.

4 S. Yamauchi, N. Hirota, S. Takahara, H. Misawa, K. Sawabe, H. Sakuragi and K. Tokumaru, J. Am. Chem. Soc., 1989, 111, 4402.

5 H. G. Korth, W. Müller, J. Lusztyk and K. U. Ingold, Angew. Chem., Int. Ed. Engl., 1989, 28, 183.

6 A. Kitamura, H. Sakuragi, M. Yoshida and K. Tokumaru, Bull. Chem. Soc. Jpn., 1980, 53, 1393.

7 M. Buback, M. Kling, M. T. Seidel, F. D. Schott, J. Schroeder and U. Steegmüller, Z. Phys. Chem., 2001, 215, 717.

8 B. Abel, J. Assmann, M. Buback, M. Kling, R. Oswald, S. Schmatz, J. Schroeder and T. Witte, J. Phys. Chem. A, 2003, $107,5157$.

9 C. Reichardt, J. Schroeder and D. Schwarzer, J. Phys. Chem. A, 2007, 111, 10111.

10 P. Hamm, R. A. Kaindl and J. Stenger, Opt. Lett., 2000, 25, 1798.

11 R. A. Kaindl, M. Wurm, K. Reimann, P. Hamm, A. M. Weiner and M. Woerner, J. Opt. Soc. Am. B, 2000, 17, 2086.
12 H. A. Staab, W. Rohr and F. Graf, Chem. Ber./Recl., 1965, 98, 1122.

13 F. Neese, ORCA - An ab initio, DFT and semiempirical SCF-MO package, Version 2.6.00, edn, University of Bonn, 2007.

14 F. Neese, J. Am. Chem. Soc., 2006, 128, 10213.

15 F. Neese, J. Comput. Chem., 2003, 24, 1740.

16 A. D. Becke, Phys. Rev. A: At., Mol., Opt. Phys., 1988, 38, 3098.

17 J. P. Perdew, Phys. Rev. B: Condens. Matter Mater. Phys., 1986, 33, 8822.

18 A. Schaefer, H. Horn and R. Ahlrichs, J. Chem. Phys., 1992, 97, 2571.

19 R. A. Kendall, T. H. Dunning, Jr. and R. J. Harrison, J. Chem. Phys., 1992, 96, 6769.

20 K. Eichkorn, O. Treutler, H. Ohm, M. Haser and R. Ahlrichs, Chem. Phys. Lett., 1995, 240, 283.

21 J. Neugebauer and B. A. Hess, J. Chem. Phys., 2003, 118, 7215.

22 M. Sax and R. K. McMullan, Acta Crystallogr., 1967, 22, 281.

23 A. V. Friderichsen, J. G. Radziszewski, M. R. Nimlos, P. R. Winter, D. C. Dayton, D. E. David and G. B. Ellison, J. Am. Chem. Soc., 2001, 123, 1977.

24 A. Kandratsenka, J. Schroeder, D. Schwarzer and V. S. Vikhrenko, Phys. Chem. Chem. Phys., 2005, 7, 1205.

25 A. Kandratsenka, J. Schroeder, D. Schwarzer and V. S. Vikhrenko, Phys. Chem. Chem. Phys., 2007, 9, 1688. 